\title{
Neutrophil Recovery Time
}

National Cancer Institute

\section{Source}

National Cancer Institute. Neutrophil Recovery Time. NCI Thesaurus. Code C158855.

The duration of time it takes for an individual who has had chemotherapy-induced neutropenia to achieve an acceptable numbers of neutrophils. 